\title{
FLAVONOIDS FROM ONOPORDON HETERACANTHUM C.A. MEY
}

\author{
S. A. Youssef
}

Department of Pharmacognosy, Faculty of Pharmacy, Assiut University, Assiut, Egypt

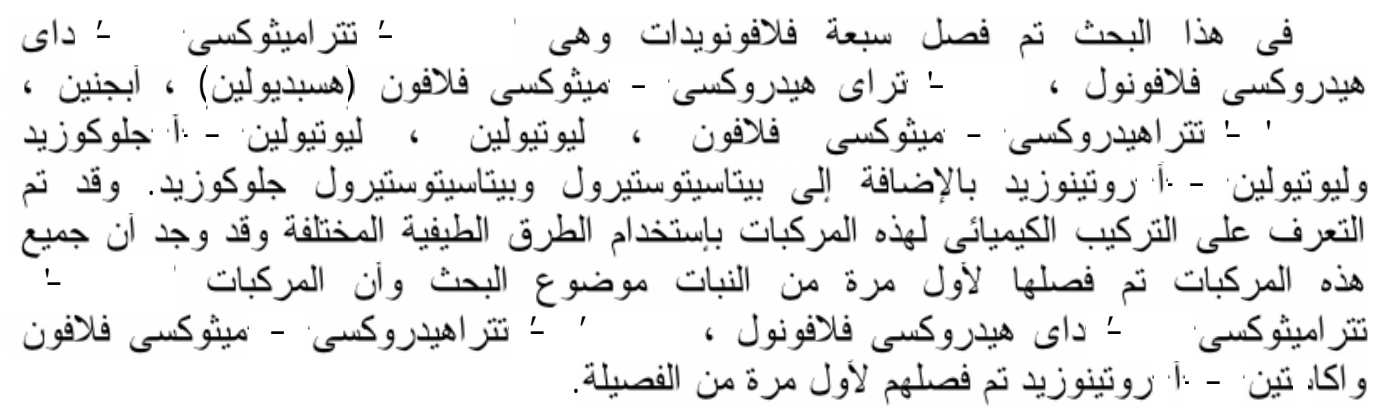

Seven flavonoids were isolated viz., 3,6,7,4'-tetramethoxy-5,3'-dihydroxy flavonol, 5,7,4'trihydroxy-6-methoxy flavone (hispedulin), apigenin, 5,7,3',4'-tetrahydroxy-6-methoxy flavone, luteolin, luteolin-7-O-glucoside, acacetin-7-O-rutinoside, in addition to $\beta$-sitosterol and $\beta$ sitosterol glucoside from Onopordon heteracanthum. The structures of these compounds were established through the chemical and spectral studies. All the isolated compounds were reported for the first time from the titled plant, while the compounds 3,6,7,4'-tetramethoxy-5,3'dihydroxy flavonol, 5,7,3'4'-tetrahydroxy-6-methoxy flavone and acacetin-7-O-rutinoside were isolated for the first time from the genus Onopordon.

\section{INTRODUCTION}

Onopordon heteracanthum C.A. Mey is a member of a small genus in the tribe Cynarea (Compositae). ${ }^{1}$ The plants of the genus Onopordon have been employed traditionally for their antibacterial, heamostatic, and antihypertensive properties ${ }^{2}$ and for treatment of face cancers since early times. $^{3}$ A pharmacological testing of onopordopigin, a sesquiterpene lactone which was isolatd from some plants belonging to the genus Onopordon, revealed anticancer activity. 4,5

Flavonoids, ${ }^{6-8}$ lignans, ${ }^{6,9}$ and sesquiterpene lactones ${ }^{10-14}$ being the most characteristic constituents of several species of the genus Onopordon.

\section{EXPERIMENTAL}

\section{Plant material}

The plant material was collected in March 2001 during the flowering stage, from El-Baha,
Southwest Saudia Arabia. Identity was confirmed by Prof. Dr. A. Fayed, Prof. of Taxonomy, Faculty of Science, Assiut University. The aerial parts were dried and powdered.

\section{Authentics}

Authentic sugars were obtained from Merck, Darmstadt, Germany, $\beta$-sitosterol, $\beta$ sitosterol glucoside, luteolin and apigenin were obtained from the Pharmacognosy Department, Faculty of Pharmacy, Assiut University.

\section{Systems}

I- Hexane - ethyl acetate

II- Hexane - ethyl acetate

III- Hexane - ethyl acetate

IV- Chloroform - methanol

V- Chloroform - methanol

VI- Ethyl acetate - methanol (80:20)

VII- n-Butanol - acetone - formic acid - water (60:17:8:15) 


\section{Equipments}

All mps are determined using Sturat Scientific apparatus and uncorrected. Mass spectra were carried out on Jeol, JMS, $600 \mathrm{H}$, NMR spectra were recorded at 400 and 100 $\mathrm{MHz}$ for ${ }^{1} \mathrm{H}$ and ${ }^{13} \mathrm{C}$ respectively in DMSO- $\mathrm{d}_{6}$ and $\mathrm{CDCl}_{3}$ using Jeol JNM-LA400. Silica gel for column (70-230 mesh), TLC using precoated silica gel sheets (E-Merck).

Flavonoidal spots were visualized by their fluorescence at $254 \mathrm{~nm}$ under UV lamp or by spraying with $\mathrm{AlCl}_{3}$. $\beta$-sitosterol spots was visualized by spraying with anisaldehyde solution and heating at $105^{\circ}$ for 10 minutes.

\section{Extraction and isolation}

The air dried aerial parts of the plant (1.0 $\mathrm{kg})$ were defatted with $\mathrm{n}$-hexane $(4 \times 1.5 \mathrm{~L})$ and successively extractd with chloroform $(5 \times 1.5$ L) and ethyl acetate $(4 \times 1.5 \mathrm{~L})$ to give 50 and 20 $\mathrm{g}$ of residue, respectively. Part of the chloroform extract residue $(20 \mathrm{~g})$ was chromatographed on a silica gel column using hexane and hexane - ethyl acetate, gradient method to give six pure compounds.

Five grams of the ethyl acetate fraction $(20 \mathrm{~g})$ was chromatographed on a silica gel column using chloroform - methanol gradient to give three pure compounds.

\section{Acid hydrolysis}

$15 \mathrm{mg}$ of each compound was refluxed with $2 \mathrm{~N}$ HCl-EtOH $(1: 1,15 \mathrm{ml})$ on a steam bath for 4 hours. The reaction mixture was diluted with water and extracted with $\mathrm{CHCl}_{3}$ $(3 \times 250 \mathrm{ml})$. The chloroformic extract was concentrated under reduced pressure. Each aglycone was purified by recrystallization from $\mathrm{CHCl}_{3}$ containing few drops of methanol. Each recovered aglycone part was identified by direct comparison with authentic samples on TLC using solvent systems II and III for flavonoid aglycones and system I for $\beta$ sitosterol. The aqueous layer was neutralized and the sugar was identified by TLC (using a solvent system VII) and comparison with authentic samples.

\section{RESULTS AND DISCUSSION}

The aerial parts of Onopordon heteracanthum afforded four flavones, one flavonol, two flavone glycosides, $\beta$-sitosterol and $\beta$-sitosterol glucoside.

Compound 1: Eluted with hexane - ethyl acetate $(9: 1)$ as white fine needles $(\mathrm{MeOH}), 84$ mg, m.p 135-137 ${ }^{\circ}, \mathrm{R}_{\mathrm{f}} 0.52$ (solvent system I). This compound was identified as $\beta$-sitosterol by direct authentication (m.m.p and cochromatography using system I).

Compound 2: Eluted with hexane - ethyl acetate $(85: 15)$ as yellow powder $(\mathrm{MeOH}), 50$ $\mathrm{mg}, \mathrm{R}_{\mathrm{f}} 0.50$ (solvent system II), m.p 228-230 . The MS (rel. int.\%): $374\left[\mathrm{M}^{+}\right]$base, 359 (79), 356 (50), 331 (69), 181 (6), 178 (13), 153 (10) and 151 (9). The UV spectral analysis of compounds 2 in methanol showed absorption at $\lambda_{\max } 346 \mathrm{~nm}$ which characteristic for 3-blocked flavonol or flavone. The shift in $\mathrm{AlCl}_{3}$, $\mathrm{AlCl}_{3} / \mathrm{HCl}$ (band I) and $\mathrm{NaOAc}$ (band II) in Table 1 indicated the absence of orthodihydroxy group in ring B (3',4'orthodihydroxy group) and absence of free $\mathrm{OH}$ at C-7 respectively. ${ }^{15}$ The ${ }^{1} \mathrm{H}-\mathrm{NMR}$ data (Table 2) revealed doublets at $\delta 7.7(\mathrm{~J}=1.7 \mathrm{~Hz})$ for $\mathrm{H}-$ $2^{\prime}$, at $7.05(\mathrm{~J}=8.5 \mathrm{~Hz})$ assigned for $\mathrm{H}-5$ ' and at $7.6(\mathrm{dd}, \mathrm{J}=8.5,1.7)$ for $\mathrm{H}-6$ ', a singlet at 6.05 (s) for $\mathrm{H}-8$ and four singlets at $\delta 3.85,3.92$, 3.95 and 3.98 (s) assigned for four methoxy groups at C-4', C-7, C-6 and C-3 respectively. The MS displayed molecular ion peak at $\mathrm{m} / \mathrm{z}$ 374 consistent with the molecular formula $\mathrm{C}_{19} \mathrm{H}_{18} \mathrm{O}_{8}$ and in accordance with flavonols with four methoxy and two hydroxy groups. The ${ }^{13} \mathrm{C}-\mathrm{NMR}$ (Table 3 ) showed that a methoxy group (downfield at $\delta$ 60.1) was flanked by ortho-oxygenated carbons. ${ }^{16}$ The other signal (downfield at $\delta$ 60.8) was attributed to a methoxy group at C-3 flavonol. ${ }^{11}$ The rest of the signals were in accord to the flavonol 3,6,7,4'-tetramethoxy-5,3'-dihydroxy flavonol. The already mentioned data indicated that compound 2 was 3,6,7,4'-tetramethoxy-5,3'dihydroxy flavonol.

Compound 3: Eluted with hexane - ethyl acetate (80:20) as yellow needles $(\mathrm{MeOH}), 40$ $\mathrm{mg}, \mathrm{R}_{\mathrm{f}} 0.46$ (solvent system II), m.p 264-267 . The MS (rel. int.\%): $300\left[\mathrm{M}^{+}\right]$(10), other characteristic fragments at 285 (67), 272 (11), 269 (8), 257 (57), 183 (10), 153 (11), 139 (19), 121 (13), 118 (33) and 69 (68). Its UV absorption data (Table 1) with different ionizing 
<smiles>[R20]Oc1ccc(-c2oc3cc([R20])c([R])c(O)c3c(=O)c2[R5])cc1[R]</smiles>

$\begin{array}{cccccc}\text { Compd. } & \mathrm{R}_{1} & \mathrm{R}_{2} & \mathrm{R}_{3} & \mathrm{R}_{4} & \mathrm{R}_{5} \\ \mathbf{2} & \mathrm{Me} & \mathrm{OMe} & \mathrm{OH} & \mathrm{Me} & \mathrm{OMe} \\ \mathbf{3} & \mathrm{H} & \mathrm{OMe} & \mathrm{H} & \mathrm{H} & \mathrm{H} \\ \mathbf{4} & \mathrm{H} & \mathrm{H} & \mathrm{H} & \mathrm{H} & \mathrm{H} \\ \mathbf{5} & \mathrm{H} & \mathrm{OMe} & \mathrm{OH} & \mathrm{H} & \mathrm{H} \\ \mathbf{6} & \mathrm{H} & \mathrm{H} & \mathrm{OH} & \mathrm{H} & \mathrm{H}\end{array}$

Table 1: UV spectrald ata of compounds $2,3,4,5,6,8$ and 9.

\begin{tabular}{|c|c|c|c|c|c|c|}
\hline Comp. No. & $\mathrm{MeOH}$ & $\mathrm{NaOMe}$ & $\mathrm{AlCl}_{3}$ & $\mathrm{AlCl}_{3} / \mathrm{HCl}$ & $\mathrm{NaOAc}$ & $\mathrm{NaOAc} / \mathrm{H}_{3} \mathrm{BO}_{3}$ \\
\hline 2 & $\begin{array}{l}253 \\
273 \\
346 \\
\end{array}$ & $\begin{array}{l}269 \\
363\end{array}$ & $\begin{array}{l}266 \\
377\end{array}$ & $\begin{array}{l}266 \\
366\end{array}$ & $\begin{array}{l}254 \\
366\end{array}$ & $\begin{array}{l}260 \\
344\end{array}$ \\
\hline 3 & $\begin{array}{l}273 \\
333\end{array}$ & $\begin{array}{c}274 \\
325(\mathrm{sh}) \\
390\end{array}$ & $\begin{array}{l}285 \\
358\end{array}$ & $\begin{array}{l}286 \\
351\end{array}$ & $\begin{array}{l}286 \\
350\end{array}$ & $\begin{array}{l}277 \\
339\end{array}$ \\
\hline 4 & $\begin{array}{l}262 \\
337\end{array}$ & $\begin{array}{c}270 \\
290(\mathrm{sh}) \\
392\end{array}$ & $\begin{array}{c}269 \\
298(\mathrm{sh}) \\
380\end{array}$ & $\begin{array}{l}270 \\
380\end{array}$ & $\begin{array}{l}272 \\
374\end{array}$ & $\begin{array}{l}267 \\
337\end{array}$ \\
\hline 5 & $\begin{array}{l}274 \\
343\end{array}$ & $\begin{array}{l}265 \\
400\end{array}$ & $\begin{array}{l}275 \\
423\end{array}$ & $\begin{array}{l}286 \\
365\end{array}$ & $\begin{array}{l}279 \\
345\end{array}$ & $\begin{array}{l}266 \\
371\end{array}$ \\
\hline 6 & $\begin{array}{l}253 \\
267 \\
350\end{array}$ & $\begin{array}{l}266 \\
330 \\
401\end{array}$ & $\begin{array}{l}273 \\
301 \\
435\end{array}$ & $\begin{array}{l}275 \\
364 \\
388\end{array}$ & $\begin{array}{l}270 \\
385\end{array}$ & $\begin{array}{l}260 \\
301 \\
380\end{array}$ \\
\hline 8 & $\begin{array}{l}270 \\
295 \\
352\end{array}$ & $\begin{array}{l}271 \\
300 \\
400\end{array}$ & $\begin{array}{c}270 \\
300(\mathrm{sh}) \\
325(\mathrm{sh}) \\
430\end{array}$ & $\begin{array}{c}271 \\
290(\mathrm{sh}) \\
360(\mathrm{sh}) \\
388\end{array}$ & $\begin{array}{c}264 \\
350(\mathrm{sh}) \\
412\end{array}$ & $\begin{array}{l}265 \\
370\end{array}$ \\
\hline 9 & $\begin{array}{l}269 \\
347\end{array}$ & $\begin{array}{l}282 \\
376\end{array}$ & $\begin{array}{c}276 \\
299(\mathrm{sh}) \\
348(\mathrm{sh}) \\
382\end{array}$ & $\begin{array}{c}276 \\
299(\mathrm{sh}) \\
338(\mathrm{sh}) \\
383\end{array}$ & $\begin{array}{l}268 \\
324\end{array}$ & $\begin{array}{l}268 \\
325\end{array}$ \\
\hline
\end{tabular}

Table 2: ${ }^{1} \mathrm{H}-\mathrm{NMR}$ spectral data of compounds $\mathbf{2 , 3}$ and $\mathbf{5}$ in DMSO-d $\mathrm{d}_{6}$.

\begin{tabular}{||c|c|c|c|c|c|c|c||}
\hline $\begin{array}{c}\text { Comp. } \\
\text { No. }\end{array}$ & H-3 & H-8 & H-2' & H-3' & H-5' & H-6' & $\mathrm{OCH}_{3}$ group \\
\hline $\mathbf{2}^{*}$ & - & $6.50 \mathrm{~s}$ & $\begin{array}{c}7.7(\mathrm{~d}, \mathrm{~J}= \\
1.7 \mathrm{~Hz})\end{array}$ & - & $\begin{array}{c}7.05(\mathrm{~d}, \mathrm{~J}= \\
8.5 \mathrm{~Hz})\end{array}$ & $\begin{array}{c}7.6(\mathrm{dd}, \mathrm{J}= \\
8.5,1.7 \mathrm{~Hz})\end{array}$ & $\begin{array}{c}3.85,3.92, \\
3.95,3.98(\mathrm{~s})\end{array}$ \\
\hline $\mathbf{3}$ & $6.5(\mathrm{~s})$ & $6.7(\mathrm{~s})$ & $\begin{array}{c}7.9(\mathrm{~d}, \mathrm{~J}= \\
8.8 \mathrm{~Hz})\end{array}$ & $\begin{array}{c}6.8(\mathrm{~d}, \mathrm{~J}= \\
8.8 \mathrm{~Hz})\end{array}$ & $\begin{array}{c}6.8(\mathrm{~d}, \mathrm{~J}= \\
8.8 \mathrm{~Hz})\end{array}$ & $\begin{array}{c}7.9(\mathrm{~d}, \mathrm{~J}= \\
8.8 \mathrm{~Hz})\end{array}$ & $3.73(\mathrm{~s})$ \\
\hline $\mathbf{5}$ & $6.5(\mathrm{~s})$ & $6.7(\mathrm{~s})$ & $7.3($ br.s) & & $\begin{array}{c}6.94(\mathrm{~d}, \mathrm{~J}= \\
8.5 \mathrm{~Hz})\end{array}$ & $\begin{array}{c}7.4(\mathrm{~d}, \mathrm{~J}= \\
8.5 \mathrm{~Hz})\end{array}$ & $3.73(\mathrm{~s})$ \\
\hline
\end{tabular}

*In $\mathrm{CDCl}_{3}$. 
Table 3: ${ }^{13} \mathrm{C}-\mathrm{NMR}$ spectral data of compounds 2, 3 and 5 (DMSO- $\mathrm{d}_{6}$ for compounds $\mathbf{3}$ and $\mathbf{5} ; \mathrm{CDCl}_{3}$ for compound 2).

\begin{tabular}{||c|c|c|c||}
\hline $\begin{array}{c}\text { Carbon } \\
\text { no. }\end{array}$ & $\mathbf{2}^{*}$ & $\mathbf{3}$ & $\mathbf{5}$ \\
\hline 2 & 155.9 & 163.8 & 163.9 \\
3 & 138.6 & 102.3 & 102.4 \\
4 & 178.8 & 182.0 & 182.0 \\
5 & 152.7 & 152.7 & 152.8 \\
6 & 132.2 & 131.3 & 131.3 \\
7 & 158.7 & 157.2 & 157.3 \\
8 & 90.3 & 94.1 & 94.1 \\
9 & 152.2 & 152.3 & 152.4 \\
10 & 106.5 & 104.0 & 104.0 \\
$1^{\prime}$ & 122.5 & 121.0 & 121.5 \\
$2^{\prime}$ & 110.8 & 128.4 & 113.3 \\
$3^{\prime}$ & 146.3 & 115.9 & 145.7 \\
$4^{\prime}$ & 148.3 & 161.0 & 149.7 \\
$5^{\prime}$ & 114.5 & 115.9 & 116.0 \\
$6^{\prime}$ & 122.4 & 128.4 & 118.9 \\
OMe & 60.8 & 59.9 & 59.9 \\
& 60.1 & & \\
\multicolumn{4}{|l}{} \\
\hline
\end{tabular}

$*$ In $\mathrm{CDCl}_{3}$.

and complexing reagent showed that the compound 3 contains free $\mathrm{OH}$ group at $\mathrm{C}-7$ (NaOAc shift, band II), and a free 4'-hydroxy group (bathochromic shift of band I in $\mathrm{NaOMe}$ with increase in intensity) and no orthodihydroxy group in $\mathrm{A}$ ring $\left(\mathrm{AlCl}_{3}, \mathrm{AlCl}_{3} / \mathrm{HCl}\right)$. The ${ }^{1} \mathrm{H}-\mathrm{NMR}$ spectrum (Table 2) showed the presence of signals at $\delta 6.7(1 \mathrm{H}, \mathrm{H}-8)$ for Aring and $6.5(1 \mathrm{H}, \mathrm{H}-3)$ for the proton $\gamma$-pyrone ring, at $\delta 6.8\left(2 \mathrm{H}, \mathrm{H}-3^{\prime}, 5^{\prime}\right), 7.9\left(2 \mathrm{H}, \mathrm{H}-2^{\prime}, 6^{\prime}\right)$ for the protons of B-ring and the singlet at $\delta 3.73$ $\left(3 \mathrm{H}, \mathrm{OCH}_{3}\right)$ was attributed to the O-methyl group at C-6'. The mass spectrum of $\mathbf{3}$ showed a peak at $\mathrm{m} / \mathrm{z} 300$ corresponding to the molecular formula $\mathrm{C}_{16} \mathrm{H}_{12} \mathrm{O}_{6}$ (DEPt, ${ }^{13} \mathrm{C}$-NMR and MS). The ${ }^{13} \mathrm{C}-\mathrm{NMR}$ (Table 3) showed that the methoxy group (downfield at $\delta$ 59.9) was flanked by ortho-oxygenated carbons (i.e. 5,7dihydroxy-6-methoxy flavone). ${ }^{16}$ The structure was moreover supported by the close resemblance of the physical and spectral data (UV, ${ }^{1} \mathrm{H}-\mathrm{NMR}$ and ${ }^{13} \mathrm{C}-\mathrm{NMR}$ ) with those reported for 5,7,4'-trihydroxy-6-methoxy flavone (hispedulin). ${ }^{17}$
Compound 4: Eluted with hexane - ethyl acetate (78:22) as yellow amorphous powder $(\mathrm{MeOH}), 15 \mathrm{mg}, \mathrm{R}_{\mathrm{f}} 0.40$ (solvent system II), m.p 343-345 . The MS (rel. int.\%): $270\left[\mathrm{M}^{+}\right]$ (23), 269 (87), 241 (70), 240 (50), 153 (77), 149 (45), 121 (74), 114 (34) and 69 (70). UV (Table 1), m.p, m.m.p and co-chromatography with authentic sample indicated that compound 4 is apigenin.

Compound 5: Eluted with hexane - ethyl acetate (60:40) as yellow needles $(\mathrm{MeOH}), 50$ $\mathrm{mg}, \mathrm{R}_{\mathrm{f}} 0.28$ (solvent system II), m.p 259-262 . The MS (rel. int.\%): $316\left[\mathrm{M}^{+}\right]$(56), 300 (40), 297 (28), 272 (49), 197 (12), 134 (60) and 69 (100). The UV spectra (Table 1) in different shift reagents indicated the presence of free $\mathrm{OH}$ at $\mathrm{C}-7$ (NaOAc), 3',4'-ortho-dihydroxy group in ring-B $\left(\mathrm{AlCl}_{3}, \mathrm{AlCl}_{3} / \mathrm{HCl}\right.$ and $\mathrm{NaOAc} /$ boric acid spectra) and 4'-OH (NaOMe, bathochromic shift with increase in intensity). The ${ }^{1} \mathrm{H}-\mathrm{NMR}$ (Table 2) showed signal at $\delta 6.5$ $(1 \mathrm{H}, \mathrm{s}, \mathrm{H}-3)$, signal for A-ring proton at $\delta 6.7$ $(1 \mathrm{H}, \mathrm{s}, \mathrm{H}-8)$, signals for B-ring protons at $\delta$ $6.94\left(1 \mathrm{H}, \mathrm{d}, \mathrm{H}-5^{\prime}\right), 7.4\left(1 \mathrm{H}, \mathrm{d}, \mathrm{H}-66^{\prime}\right), 7.3(1 \mathrm{H}$, br.s, $\mathrm{H}-2$ ') and $3.73\left(3 \mathrm{H}, \mathrm{s}, \mathrm{OCH}_{3}\right.$ at $\left.\mathrm{C}-6\right)$. The mass spectrum showed a peak at $\mathrm{m} / \mathrm{z} 316$ corresponding to the molecular formula $\mathrm{C}_{16} \mathrm{H}_{12} \mathrm{O}_{7}$. The ${ }^{13} \mathrm{C}-\mathrm{NMR}$ (Table 3) showed a characteristic downfield shift signal at $\delta 59.9$ for methoxy group at C- 6 between two orthooxygenated carbon (i.e. 5,7-dihydroxy-6methoxy flavone). The downfield shift of C-4 at $\delta 182.0$ as in compound $\mathbf{3}$ indicated the presence of hydroxy at C-5 in flavone. ${ }^{16}$

The above mentioned data suggest the flavonoid $\mathbf{5}$ is 5,7,3',4'-tetrahydroxy-6-methoxy flavone. The structure of compound $\mathbf{5}$ was moreover supported by the close resemblance of the ${ }^{13} \mathrm{C}-\mathrm{NMR}$ spectral data with those reported for 5,7,3',4'-tetrahydroxy-6-methoxy flavone. ${ }^{18}$

Compound 6: Eluted with hexane - ethyl acetate (10:90) as yellow amorphous powder $(\mathrm{MeOH}), 18 \mathrm{mg}, \mathrm{R}_{\mathrm{f}} 0.23$ (solvent system III), m.p 328-330 . MS m/z (rel. int.\%): 286 (100), 273 (22), 258 (18), 229 (8) and 203 (6). UV (Table 1), m.p, m.m.p and co-chromatography with authentic sample indicated that compound $\mathbf{6}$ is luelolin. 
Compound 7: Eluted with chloroform methanol (90:10) as white powder, $400 \mathrm{mg}, \mathrm{R}_{\mathrm{f}}$ 0.44 (solvent system IV), m.p $278-279^{\circ}$. It was identified as $\beta$-sitosterol glucoside by direct authentication (m.p, m.m.p, cochromatography) as well as the aglycone $\beta$ sitosterol upon acid hydrolysis (see experimental part).

Compound 8: Eluted with chloroform methanol $(85: 15)$ as yellow amorphous powder $(\mathrm{MeOH}), 150 \mathrm{mg}, \mathrm{R}_{\mathrm{f}} 0.58$ (solvent system $\mathrm{V}$ ), m.p 255-258 ${ }^{\circ}$. Its UV data (Table 1) indicated that compound $\mathbf{8}$ is a flavone with $3^{\prime}, 4^{\prime}$-orthodihydroxy group $\left(\mathrm{AlCl}_{3}, \mathrm{AlCl}_{3} / \mathrm{HCl}\right.$ and $\mathrm{NaOAc} / \mathrm{H}_{3} \mathrm{BO}_{3}$ shift) and absence of $\mathrm{OH}$ group at C-7 (NaOAc shift). The ${ }^{1} \mathrm{H}-\mathrm{NMR}$ spectral data (Table 4) showed signals for $\gamma$-pyrone ring at $\delta 6.7(\mathrm{~s}, \mathrm{H}-3)$, for A-ring at $\delta 6.4(\mathrm{~d}, \mathrm{H}-6)$, $6.8(\mathrm{~d}, \mathrm{H}-8)$, for B-ring at $\delta 7.0\left(\mathrm{~d}, \mathrm{H}-5^{\prime}\right), 7.5$ (dd, H-6'), 7.4 (d, H-2'). Anomeric proton of glucose appeared at $\delta 5.1(1 \mathrm{H}, \mathrm{d})$. Acid hydrolysis (see experimental) gave aglycone whose data are identical for luteolin and the sugar was found to be glucose (cochromatography with authentic sample).

From the previous data and comparing those data with the published data for luteolin7-O-glucoside, ${ }^{15,19}$ so compound $\mathbf{8}$ was identified as luteolin-7-O-glucoside. The above data was confirmed by ${ }^{13} \mathrm{C}$-NMR data (Table $5)$.
Compound 9: Eluted with chloroform methanol (80:20) as yellow amorphous powder $(\mathrm{MeOH}), 90 \mathrm{mg}, \quad \mathrm{m} . \mathrm{p} 238-240^{\circ}, \mathrm{R}_{\mathrm{f}} 0.50$ (Solvent system VI). The UV data (Table 1) showed that compound 9 contains no free $\mathrm{OH}$ at C-4', C-7 and absence of ortho-dihydroxy group from the $\mathrm{NaOMe}, \mathrm{NaOAc}$ and $\mathrm{AlCl}_{3}$, $\mathrm{AlCl}_{3} / \mathrm{HCl}$ respectively. The ${ }^{1} \mathrm{H}-\mathrm{NMR}$ spectrum (Table 4) showed aromatic protons of C-2' and of C-6' appeared at $\delta 8.03(\mathrm{~d}, 2 \mathrm{H})$, protons of C-3' and C-5' at $\delta 7.1(\mathrm{~d}, 2 \mathrm{H})$, proton of $\mathrm{C}-3$ at $\delta 6.7(\mathrm{~s}, 1 \mathrm{H})$, proton at $\delta 6.4(1 \mathrm{H}$, br.s) for $\mathrm{C}-6$ and at $\delta 6.9(1 \mathrm{H}$, br.s) for $\mathrm{C}-8$, anomeric proton of glucose appeared at $\delta 5.1(1 \mathrm{H}, \mathrm{d})$ and that for rhamnose at $\delta 4.5\left(1 \mathrm{H}\right.$, br.s) and $\mathrm{CH}_{3}$ for rhamnose at $\delta 1.07(1 \mathrm{H}, \mathrm{d})$. The methoxy protons appeared at $\delta 3.84(3 \mathrm{H}, \mathrm{s})$. Acid hydrolysis (see experimental) gave aglycone, whose identical to acacetin (cochromatography, m.p and m.m.p with authenic sample), and the sugars by using TLC chromatography gave spots of glucose and rhamnose when compared with authentic sugars.

From the previous data and comparing with the published data ${ }^{20}$ the compound 9 was suggested to be acacetin-7-O-rutinoside which confirmed by ${ }^{13} \mathrm{C}-\mathrm{NMR}$ data (Table 5).<smiles>[R20]c1cc(O)c2c(=O)cc(-c3ccc([R20])c([R3])c3)oc2c1</smiles>

$\begin{array}{cccc}\text { Compd. } & \mathrm{R}_{1} & \mathrm{R}_{2} & \mathrm{R}_{3} \\ \mathbf{8} & \text { glucose } & \mathrm{H} & \mathrm{OH} \\ \mathbf{9} & \text { glucose-rhamnose } & \mathrm{CH}_{3} & \mathrm{H}\end{array}$


Table 4: ${ }^{1} \mathrm{H}-\mathrm{NMR}$ spectral data of compounds 8 and 9 in DMSO- $\mathrm{d}_{6}$.

\begin{tabular}{|c|c|c|c|c|c|c|c|}
\hline $\begin{array}{c}\text { Comp. } \\
\text { No. }\end{array}$ & H-3 & H-6 & H-8 & $\begin{array}{c}\text { H-2' } \\
\text { (comp. 8) }\end{array}$ & $\begin{array}{c}\text { H-5' } \\
\text { (comp. 8) } \\
\text { H-3',5' } \\
\text { (comp. 9) }\end{array}$ & $\begin{array}{c}\text { H-6' (comp. } \\
\mathbf{8}) \\
\text { H-2',6' } \\
\text { (comp. 9) }\end{array}$ & $\begin{array}{l}\mathrm{OCH}_{3} \\
\text { group }\end{array}$ \\
\hline 8 & $6.7(\mathrm{~s})$ & $\begin{array}{c}6.4(\mathrm{~d}, \mathrm{~J}= \\
1.9 \mathrm{~Hz})\end{array}$ & $\begin{array}{c}6.8(\mathrm{~d}, \mathrm{~J}= \\
1.9 \mathrm{~Hz})\end{array}$ & $\begin{array}{c}7.4(\mathrm{~d}, \mathrm{~J}= \\
2.1 \mathrm{~Hz})\end{array}$ & $\begin{array}{c}7.0(\mathrm{~d}, \mathrm{~J}= \\
8.5 \mathrm{~Hz})\end{array}$ & $\begin{array}{c}7.5(\mathrm{dd}, \mathrm{J}= \\
8.5,2.1 \mathrm{~Hz})\end{array}$ & - \\
\hline 9 & $6.7(\mathrm{~s})$ & 6.4 (br.s) & 6.9 (br.s) & - & $\begin{array}{c}7.1(\mathrm{~d}, \mathrm{~J}= \\
8.5 \mathrm{~Hz})\end{array}$ & $\begin{array}{c}8.03(\mathrm{~d}, \mathrm{~J}= \\
8.5 \mathrm{~Hz})\end{array}$ & $3.84(\mathrm{~s})$ \\
\hline
\end{tabular}

The sugar protons:

a) Compound $\mathbf{8}$ shows signal at $\delta 5.1(\mathrm{~d}, \mathrm{~J}=7.3 \mathrm{~Hz})$ for anomeric proton of glucose.

b) Ccompound 9 shows signal at $\delta 5.06(\mathrm{~d}, \mathrm{~J}=7.08 \mathrm{~Hz})$ for anomeric proton of glucose. Signal at $\delta$ 4.5 (br.s) for anomeric proton of rhamnose and signal at $\delta 1.07(\mathrm{~d}, \mathrm{~J}=6.1 \mathrm{~Hz})$ assigned for $\mathrm{CH}_{3}$ of rhamnose.

Table 5: ${ }^{13} \mathrm{C}-\mathrm{NMR}$ spectral data of compounds 8 and $\mathbf{9}\left(\mathrm{DMSO}_{\mathrm{-}} \mathrm{d}_{6}\right)$.

\begin{tabular}{|c|c|c|}
\hline Carbon no. & 8 & 9 \\
\hline 2 & 164.5 & 164.0 \\
\hline 3 & 103.2 & 103.8 \\
\hline 4 & 181.9 & 181.4 \\
\hline 5 & 161.1 & 161.1 \\
\hline 6 & 99.9 & 99.9 \\
\hline 7 & 162.9 & 162.9 \\
\hline 8 & 94.7 & 94.8 \\
\hline 9 & 156.9 & 157.0 \\
\hline 10 & 105.4 & 105.4 \\
\hline $1^{\prime}$ & 121.4 & 122.6 \\
\hline $2^{\prime}$ & 113.5 & 114.7 \\
\hline $3^{\prime}$ & 145.8 & 128.5 \\
\hline $4^{\prime}$ & 149.9 & 162.4 \\
\hline $5^{\prime}$ & 116.0 & 128.5 \\
\hline $6^{\prime}$ & 119.2 & 114.7 \\
\hline $\mathrm{OMe}$ & - & 55.6 \\
\hline 1" & 99.5 & 99.7 \\
\hline $2^{\prime \prime}$ & 73.1 & 73.1 \\
\hline 3" & 76.4 & 76.3 \\
\hline 4" & 69.5 & 69.6 \\
\hline $5^{\prime \prime}$ & 77.2 & 77.1 \\
\hline 6" & 60.6 & 68.3 \\
\hline $1^{\prime \prime \prime}$ & - & 100.5 \\
\hline $2^{\prime \prime \prime}$ & - & 70.3 \\
\hline $3^{\prime \prime \prime}$ & - & 70.7 \\
\hline $4^{\prime \prime \prime}$ & - & 72.0 \\
\hline $5^{\prime \prime \prime}$ & - & 75.7 \\
\hline 6"' & - & 17.8 \\
\hline
\end{tabular}

\section{Acknowledgment}

The author wish to thank Prof. Abdel-Aziz Fayed, Prof. of Taxonomy, Faculty of Science, Assiut University for supplying the plant material.

\section{REFERENCES}

1- T. G. Tutin, V. H. Heywood, N. A. Burger, D. M. Moore, D. H. Valentine, S. M. Walters and D. A. Webbe, "Flora Europaea", Cambridge University Press Cambridge, Vol. 4, p. 246 (1976).

2- E. Maugini, "Manuale di Botanica Farmaceutica, Piccin: Padova", 7th. Ed., p. 467 (1994).

3- G. Negri, "Erbario Figurato, Hoepli: Milano", p. 421 (1943).

4- G. Madaus, "Lchrbuch der Biologischen Heihmittel", Vol. 3,5, 2029, George OLMS Verlage, Hildesheim, New York (1979).

5- O. Sticher, In: H. Wagner, P. Wolff, "New Natural Products and Plant Drugs with Pharmacological, Biological and Therapeutical Activity", Springer Verlage, Berlin, p. 137 (1977).

6- L. M. Cardona, R. A. Aleman, B. Garcia and J. R. Pedro, Phytochemistry, 31, 36303632 (1992).

7- L. M. Cardona, M. I. Fernandez, J. R. Pedro and A. A. Valle, Planta Medica, 53, 506 (1987). 
8- L. M. Cardona, B. Garcia, J. R. Pedro and J. F. Sinisterra, Physochemistry, 29 (2), 629-631 (1990).

9- D. Lazari, B. Garcia, H. Skaltsa, J. R. Pedro and C. Harvala, Phytochemistry, 47, 415-422 (1998).

10- K. Y. Orabi, K. A. El-Sayed, M. S. AlSaid and F. S. El-Feraly, Saudi Pharm. J., 8 (2-3), 101-105 (2000).

11- L. M. Cardona, R. A. Bardon, B. Garcia and J. R. Pedro, Phytochemistry, 33, 14571460 (1993).

12- D. Lazari, B. Garcia, H. Skaltsa, J. R. Pedro and C. Harvala, Phytochemistry, 47, 415-422 (1997).

13- B. Garcia, H. Skaltsa, F. I. Navarro, J. R. Pedro and D. Lazari, Phytochemistry, 41, 1113-1117 (1996).

14- T. S. El-Alfy, S. El-Dahmy, M. A. Koheil, A. H. Shehat and F. Eid, Bulletin of Faculty of Pharmacy, Cairo University, 32, 109-112 (1994).
15- T. J. Mabry, K. R. Markham and M. B. Thomas, "The Systematic Identification of Flavonoids", Springer, Heidelberg (1970).

16- K. P. Agrawal, "Carbon-13-NMR of Flavonoids", Amesterdam, Oxford, New York, Tokyo, p. 20 (1989).

17- I. Gonzalez, F. A. Coliado, M. G. Macias, Massanet and F. Rodriguezluij, Journal of Natural Products, 48 (5), 819 (1985).

18- M. Mendez, A. C. Rojos, A. Bahsas, R. Jaimes and J. Triana, Acta Gient Venez, 31, 394 (1980).

19- B. J. Harborne and J. T., Mabry, "The Flavonoids: Advances in Research", London, New York, p. 73 (1982).

20- J. B. Harborne, T. J. Mabry and H. Mabry, "The Flavonoids", Chapman and Hall, London, 84 (1975). 